\title{
Hydroxyurea is associated with lower prevalence of albuminuria in adults with sickle cell disease
}

\author{
Louis-Philippe Laurin ${ }^{1}$, Patrick H. Nachman ${ }^{1}$, Payal C. Desai ${ }^{2}$, Kenneth I. Ataga ${ }^{3}$ and Vimal K. Derebail ${ }^{1}$ \\ ${ }^{1}$ UNC Kidney Center, Division of Nephrology and Hypertension, Department of Medicine, University of North Carolina at Chapel Hill, Chapel \\ Hill, NC, USA, ${ }^{2}$ Division of Hematology, Department of Medicine, The Ohio State University College of Medicine, Columbus, OH, USA and \\ ${ }^{3}$ Division of Hematology and Oncology, Department of Medicine, University of North Carolina at Chapel Hill, Chapel Hill, NC, USA
}

Correspondence and offprint requests to: Vimal K. Derebail; E-mail: vimal_derebail@med.unc.edu

\section{ABSTRACT}

Background. Albuminuria is an early manifestation of sickle cell nephropathy. Prior small case series suggests benefit of hydroxyurea in reducing albuminuria, with a similar trend noted in pediatric studies. We aimed to comprehensively evaluate hydroxyurea use and prevalence of albuminuria in adult sickle cell patients.

Methods. We performed a cross-sectional study of 149 adult patients followed between 2000 and 2011 in a comprehensive sickle cell clinic. All patients were assessed for albuminuria either by direct measurement or by urinary chemical strip (dipstick) testing. Urinary albumin-to-creatinine ratios (UACRs) were available for 112 patients. Hydroxyurea exposure was defined as $\geq 3$ months of therapy before the assessment of albuminuria. Albuminuria was defined as either UACR $\geq 30 \mathrm{mg} / \mathrm{g}$ or $\geq 1+$ proteinuria on two separate dipsticks. We constructed a multivariate logistic regression model to assess the association between hydroxyurea and albuminuria.

Results. The prevalence of albuminuria was lower among patients on hydroxyurea ( 34.7 versus $55.4 \% ; \mathrm{P}=0.01$ ) as was median albumin excretion (17.9 versus $40.5 \mathrm{mg} / \mathrm{g}$; $\mathrm{P}=0.04$ ). In multivariate analysis, hydroxyurea was associated with a lower likelihood of albuminuria (odds ratio $0.28,95 \% \mathrm{CI}$ : $0.11-0.75, \mathrm{P}=0.01$ ), adjusting for age, angiotensin-converting enzyme inhibitor/angiotensin receptor blocker use, tricuspid regurgitant jet velocity, hypertension and acute chest syndrome.

Conclusions. In our population of sickle cell patients, those using hydroxyurea were less than one-third as likely to exhibit albuminuria. Hydroxyurea use may prevent development of overt nephropathy or the progression of sickle cell disease nephropathy to end-stage renal disease, and its use for this indication merits further investigation.
Keywords: albuminuria, chronic kidney disease, hydroxyurea, nephropathy, sickle cell

\section{INTRODUCTION}

Sickle cell disease (SCD) nephropathy is a common complication of SCD, and is marked in part by repetitive vaso-occlusive phenomena leading to ischemic injury to glomeruli and the medullary vasa recta [1]. Ischemic renal damage leads to decreased urinary concentrating ability, renal tubular acidosis and glomerular ischemia with secondary hyperfiltration mediated by vasodilatory prostaglandins $[2,3]$. Histologically, SCD nephropathy is characterized by glomerular enlargement and perihilar focal segmental glomerulosclerosis (FSGS) [4-6].

Microalbuminuria is an early detectable manifestation of SCD nephropathy and occurs in $\sim 32-42 \%$ of adult SCD patients, but can vary depending on age and SCD genotype $[6,7]$. Microalbuminuria assessment is a sensitive way of detecting glomerular injury caused by SCD before appearance of a decline in glomerular filtration rate [7]. Overt proteinuria may occur in $40 \%$ of older adults and has been associated with progressive loss of renal function [5-7]. Approximately $4.2-11.6 \%$ of sickle cell anemia patients develop end-stage renal disease, which predicts early mortality $[4,8,9]$.

Few options are available for the treatment of SCD nephropathy. Based upon short-term studies, angiotensin-converting enzyme inhibitors (ACEi) may reduce overt proteinuria and remain the mainstay of therapy $[4,5,10,11]$. Hydroxyurea is known to reduce the frequency of acute painful episodes, acute chest syndrome and red blood cell transfusion requirement [12]. Hydroxyurea also appears to reduce mortality of patients with severe SCD [13-15]. Multiple small case series have suggested a short-term benefit of hydroxyurea to reduce proteinuria, and a similar trend has been noted in retrospective and prospective pediatric studies [16-19]. 
We sought to analyze the association of hydroxyurea treatment with albuminuria in a cohort of adult patients with SCD. We hypothesized that the use of hydroxyurea is associated with decreased frequency and severity of proteinuria after controlling for other factors that affect protein excretion.

\section{MATERIALS AND METHODS}

\section{Patient population}

Patients with SCD aged 18 years or older identified between 2000 and 2011 and followed at the University of North Carolina at Chapel Hill were included in this cross-sectional study. Some patients were recruited as part of a prior study to evaluate the pathophysiology of pulmonary hypertension in SCD [20]. For inclusion in our analysis, patients had to have a measure of albuminuria either by direct quantification or urinary chemical strip (dipstick) testing. Patients on renal replacement therapy were excluded. Samples used for data analysis were collected during routine outpatient clinic visits. Urine albumin measurements were taken at a time when patients were without acute illness, such as a pain crisis, infection or gross hematuria. All other clinical and laboratory variables were obtained by retrospective review of the computerized medical records. All aspects of the study were approved by our institutional review board, in agreement with the Declaration of Helsinki.

\section{Laboratory parameters}

Urinary albumin and urinary creatinine as well as other laboratory parameters were measured in the clinical laboratories of the University of North Carolina Hospitals. Albuminuria was defined as at least one urinary albumin-to-creatinine ratio (UACR) $\geq 30 \mathrm{mg} / \mathrm{g}$ or $\geq 1+$ proteinuria on two dipstick measurements at least 2 months apart. In our laboratory, urinary chemical strip testing with results $\geq 1+$ correlate with urinary protein concentrations $\geq 30 \mathrm{mg} / \mathrm{dL}$ which have $\sim 58 \%$ sensitivity but $>95 \%$ specificity for detecting UACR $\geq 30 \mathrm{mg} / \mathrm{g}$ [21, 22]. This level of dipstick positivity has been used in prior studies to identify proteinuria in sickle cell subjects [23]. For those with UACR available, albuminuria was further categorized as microalbuminuria, defined as UACR ranging from $\geq 30$ to $299 \mathrm{mg} / \mathrm{g}$, and macroalbuminuria, defined as UACR $\geq 300 \mathrm{mg} / \mathrm{g}$. The estimated glomerular filtration rate (eGFR) was calculated using the six-variable Modification of Diet in Renal Disease (MDRD) Study Group formula, with correction for the body surface area and serum albumin level [24]. Hydroxyurea treatment was defined as $\geq 3$ continuous months of therapy prior to the time of albuminuria assessment. The usual indications to start hydroxyurea were frequent pain episodes, typically more than two to three episodes requiring medical attention per year, frequent or severe episodes of acute chest and occasionally severe anemia related to SCD. A diagnosis of systemic hypertension was made if repeated arterial blood pressure values justified use of anti-hypertensive medication. Tricuspid regurgitant jet velocity (TRV) was assessed by transthoracic Doppler echocardiography. With the modified Bernoulli equation, the TRV provides a calculated estimation of the right-ventricular-to-right-atrial systolic pressure gradient $\left(\Delta \mathrm{P}=4 \times \mathrm{TRV}^{2}\right)$ [25]. Pulmonary artery systolic pressure was estimated by adding estimated mean atrial pressure to $\Delta \mathrm{P}$. While not explicitly diagnostic, an estimated TRV of $2.5 \mathrm{~m} / \mathrm{s}$ typically reflects pressures found in the mild-moderate pulmonary hypertension range [26].

\section{Statistical analyses}

Continuous variables with normal distribution were expressed as mean \pm standard deviation and compared using Student's $t$-tests. Non-parametric variables were expressed as median [inter-quartile range (IQR)] and compared with the Mann-Whitney $U$ test or Kruskal-Wallis test. The Chi-square or Fisher's exact test was used to compare all categorical variables with the presence of albuminuria, microalbuminuria and macroalbuminuria as well as hydroxyurea use.

In univariate analyses, primary outcome variables were defined as the presence of albuminuria, microalbuminuria or macroalbuminuria. For multivariate analysis, we constructed a binary logistic regression model with albuminuria as the outcome. We chose potential covariates identified in univariate analyses as well as those felt to be likely to influence the outcome as determined by directed acyclic graphs [27]. We employed backward elimination to create the most parsimonious model. Covariates were retained in the full model if their removal produced a $>10 \%$ change in the effect estimate. Notably, we chose not to include markers of hemolysis in our initial modeling as we felt these were at least in part causal intermediates of the potential association between hydroxyurea and albuminuria. Hydroxyurea may reduce erythrocyte sickling and hemolysis that may be directly responsible for renal injury. Inclusion in multivariate modeling of such a covariate could remove the detectability of an association between the primary exposure (hydroxyurea) and the outcome (albuminuria) [27]. A P-value $<0.05$ was considered as statistically significant. Confidence intervals included $95 \%$ of predicted values. Statistical analyses were performed using SPSS 16.0 (IBM Corporation, USA) software and Stata 12.1 (StataCorp LP, USA). All authors had access to the primary data; L.-P.L. and V.K.D. analyzed the data set.

\section{RESULTS}

\section{Patient characteristics}

A total of 149 patients were identified (95 females and 54 males), with a median age of 37 years (range from 18 to 71 years) (Table 1). The majority of patients had the HbSS genotype (113 $\mathrm{HbSS}, 10 \mathrm{HbS}^{0}, 7 \mathrm{HbS} \beta^{+}$and $18 \mathrm{HbSC}$ ). Hydroxyurea was used for at least 3 months (mean length of treatment of $73 \pm 53$ months) in 75 patients $(50.3 \%$ of the cohort), with a mean daily dose of $1207 \pm 382 \mathrm{mg}$. Patients were started on hydroxyurea at a median age of 27 years (IQR 21-35 years). A history of acute chest syndrome was recorded in $123(82.6 \%)$ patients. Forty-five of 95 patients $(47.4 \%)$ tested by echocardiography had a TRV $\geq 2.5 \mathrm{~m} / \mathrm{s}$. Forty $(26.8 \%)$ patients carried a diagnosis of systemic hypertension, 8 (5.4\%) had diabetes, $24(16.1 \%)$ were on an ACEi or angiotensin 
Table 1. Patient characteristics of 149 adults with sickle cell disease

\begin{tabular}{|c|c|c|}
\hline & \multicolumn{2}{|l|}{$n$} \\
\hline \multicolumn{3}{|l|}{ Clinical parameters } \\
\hline Age (years) & 149 & $37(28-48)$ \\
\hline $\operatorname{Sex}(\%)$ & 149 & \\
\hline $\mathrm{F}$ & & 63.8 \\
\hline $\mathrm{M}$ & & 36.2 \\
\hline Genotype (\%) & 149 & \\
\hline HbSS & & 75.8 \\
\hline $\mathrm{HbS} \beta^{0}$ & & 6.7 \\
\hline $\mathrm{HbS} \beta^{+}$ & & 4.7 \\
\hline $\mathrm{HbSC}$ & & 12.1 \\
\hline $\mathrm{HbSD}$ & & 0.7 \\
\hline Hydroxyurea use (\%) & 149 & 50.3 \\
\hline ACEi/ARB use (\%) & 149 & 16.1 \\
\hline NSAID use (\%) & 149 & 37.6 \\
\hline Hypertension (\%) & 149 & 26.8 \\
\hline Systolic blood pressure (mmHg) & 149 & $122 \pm 16$ \\
\hline Diastolic blood pressure (mmHg) & 149 & $70 \pm 12$ \\
\hline Diabetes (\%) & 149 & 5.4 \\
\hline $\mathrm{TRV} \geq 2.5 \mathrm{~m} / \mathrm{s}(\%)$ & 95 & 47.4 \\
\hline Acute chest syndrome (\%) & 149 & 82.6 \\
\hline \multicolumn{3}{|l|}{ Laboratory parameters } \\
\hline UACR (mg/g) & 112 & $30.2(6.2-81.7)$ \\
\hline Creatinine $(\mu \mathrm{mol} / \mathrm{L})$ & 149 & $62(53-80)$ \\
\hline eGFR by MDRD equation ( $\mathrm{mL} / \mathrm{min})$ & 140 & $140 \pm 57$ \\
\hline $\operatorname{TRV}(\mathrm{m} / \mathrm{s})$ & 95 & $2.5(2.2-2.9)$ \\
\hline Hemoglobin (g/L) & 149 & $91 \pm 18$ \\
\hline Percentage reticulocyte (\%) & 147 & $6.3(4.3-8.9)$ \\
\hline Fetal hemoglobin (\%) & 138 & $5.9(2.9-12.8)$ \\
\hline Lactate dehydrogenase (U/L) & 139 & $853(668-1159)$ \\
\hline Total bilirubin level $(\mu \mathrm{mol} / \mathrm{L})$ & 141 & $32(17-50)$ \\
\hline
\end{tabular}

Parametric continuous variables expressed as mean \pm standard deviation; non-parametric continuous variables expressed as median (inter-quartile range).

$\mathrm{ACEi}$, angiotensin-converting enzyme inhibitor; $\mathrm{ARB}$, angiotensin receptor blocker NSAID, non-steroidal anti-inflammatory drug; TRV, tricuspid regurgitation jet velocity; UACR, urinary albumin-to-creatinine ratio; eGFR, estimated glomerular filtration rate; MDRD, Modification of Diet in Renal Disease.

receptor blocker (ARB) and $56(37.6 \%)$ used non-steroidal antiinflammatory drugs at albuminuria quantification.

\section{Albuminuria}

The prevalence of albuminuria of any degree as measured either by UACR or dipstick was $45.0 \%$ (67 of 149 patients). The median UACR was $80.2 \mathrm{mg} / \mathrm{g}$ (IQR: $41.4-359.4 \mathrm{mg} / \mathrm{g}$ ) in the albuminuria group compared with $6.0 \mathrm{mg} / \mathrm{g}$ (IQR: $0-15.8 \mathrm{mg} / \mathrm{g}$ ) among those with normal albumin excretion ( $\mathrm{P}$ $<0.001$ ) (Table 2). Microalbuminuria and macroalbuminuria assessment was performed in 112 patients with documented UACR but not in patients with documented dipstick only. When categorized by severity, microalbuminuria (UACR from 30 to $299 \mathrm{mg} / \mathrm{g}$ ) was present in $34.8 \%$ of patients (39 of 112) with albumin excretion quantification, whereas macroalbuminuria (UACR $\geq 300 \mathrm{mg} / \mathrm{g}$ ) was less prevalent (16.1\%).

The presence of albuminuria was associated with lower hemoglobin $(88 \pm 18$ versus $93 \pm 17 \mathrm{~g} / \mathrm{L} ; \mathrm{P}=0.05)$, greater reticulocyte percentage $(7.0$ versus $5.5 \% ; \mathrm{P}=0.009)$ and a trend toward higher lactate dehydrogenase [932 U/L (IQR: 681$1364 \mathrm{U} / \mathrm{L}$ ) versus $802 \mathrm{U} / \mathrm{L}$ (IQR: 652-1104 U/L); P = 0.06] and total bilirubin levels [38 $\mu \mathrm{mol} / \mathrm{L}$ (IQR: $19-51 \mu \mathrm{mol} / \mathrm{L}$ ) versus
$29 \mu \mathrm{mol} / \mathrm{L}$ (IQR: $17-44 \mu \mathrm{mol} / \mathrm{L}) ; \mathrm{P}=0.06$ ], likely reflecting increased hemolysis. Patients with albuminuria were prescribed an ACEi/ARB more frequently (25.4 versus $8.5 \%$; $\mathrm{P}=0.005$ ), but received hydroxyurea less frequently (38.8 versus $59.8 \%$; $\mathrm{P}$ $=0.01$ ).

Within categories of albuminuria (microalbuminuria versus macroalbuminuria), several variables associated with severity of SCD were related to severity of albuminuria (Supplementary data Table S1). Markers of hemolysis, including low hemoglobin, high percentage of reticulocytes, high lactate dehydrogenase and elevated total bilirubin, were all associated with a worsening level of albuminuria in univariate analyses. In addition, TRV $\geq 2.5 \mathrm{~m} / \mathrm{s}$ and increased creatinine were significant univariate correlates of elevated albuminuria. Fifteen (38.5\%) patients with microalbuminuria and six (33.3\%) with macroalbuminuria were on hydroxyurea therapy, compared with $34(61.8 \%)$ who demonstrated no albuminuria during the observation period $(\mathrm{P}=0.03)$.

\section{Hydroxyurea therapy}

Hydroxyurea use was associated with a lower overall prevalence of albuminuria (34.7 versus $55.4 \% ; \mathrm{P}=0.01$ ) and a lower median UACR [17.9 mg/g (IQR: $6.0-53.0 \mathrm{mg} / \mathrm{g}$ ) versus 40.5 mg/g (IQR: 7.0-204.9 mg/g); P = 0.04] (Table 3). Notably, patients on hydroxyurea more commonly had a history of acute chest syndrome history ( 94.7 versus $70.3 \%$; $\mathrm{P}<0.001$ ), but had lower TRV by echocardiography [ $2.3 \mathrm{~m} / \mathrm{s}$ (IQR: $2.1-2.7 \mathrm{~m} / \mathrm{s}$ ) versus $2.5 \mathrm{~m} / \mathrm{s}$ (IQR: $2.3-3.0 \mathrm{~m} / \mathrm{s}$ ); $\mathrm{P}=0.009$ ]. Hydroxyurea therapy was not associated with less hemolysis, but patients not on hydroxyurea with albuminuria had a significant increase in hemolysis markers (Supplementary data Table S2). In addition, patients on hydroxyurea demonstrated relative glomerular hyperfiltration compared with those not on such treatment with the mean eGFR value of $151 \pm 55$ versus $128 \pm 58 \mathrm{~mL} / \mathrm{min}, \mathrm{P}=0.02$. When evaluating categories of albuminuria, there was a trend toward a lower prevalence of microalbuminuria and macroalbuminuria in patients on hydroxyurea that did not reach statistical significance. In univariate regression analysis, hydroxyurea therapy was associated with a reduced likelihood of having albuminuria [odds ratio (OR) 0.43, 95\% confidence interval (CI) 0.221-0.827; P = 0.01] (Table 4).

In our multivariate logistic regression model, the likelihood of having albuminuria was controlled for potential contributors to albuminuria such as age, ACEi/ARB use, TRV, hypertension and history of acute chest syndrome. After adjusting for these variables, hydroxyurea therapy remained associated with a lower risk of albuminuria (OR: 0.28, 95\% CI: 0.105-0.751; $\mathrm{P}=0.01$ ) (Table 5). Simplification of this model via backward elimination and change-in-estimate testing led to removal of age and hypertension from the model with a similar effect size (OR: 0.31, 95\% CI: 0.12-0.80; P =0.02). We also constructed a model in which a marker of hemolysis (lactate dehydrogenase) was included. There was a persistent trend toward a decreased risk of albuminuria with hydroxyurea therapy that did not reach statistical significance (OR: 0.42, 95\% CI: 0.15-1.18; $\mathrm{P}=0.1$ ). 


\begin{tabular}{|c|c|c|c|c|c|}
\hline & $n$ & Albuminuria & $n$ & No albuminuria & P-value \\
\hline Age (years) & 67 & $38(29-48)$ & 82 & $37(25-48)$ & 0.5 \\
\hline Female & & 56.7 & & 69.5 & \\
\hline Male & & 43.3 & & 30.5 & \\
\hline Genotype (\%) & 67 & & 82 & & 0.07 \\
\hline $\mathrm{HbSS}$ & & 85.1 & & 68.3 & \\
\hline $\mathrm{HbSC}$ & & 7.5 & & 15.9 & \\
\hline $\mathrm{HbSD}$ & & 1.5 & & 0 & \\
\hline Hydroxyurea use (\%) & 67 & 38.8 & 82 & 59.8 & 0.01 \\
\hline ACEi/ARB use (\%) & 67 & 25.4 & 82 & 8.5 & 0.005 \\
\hline NSAID use (\%) & 67 & 31.3 & 82 & 42.7 & 0.2 \\
\hline Hypertension (\%) & 67 & 31.3 & 82 & 23.2 & 0.3 \\
\hline Acute chest syndrome (\%) & 67 & 83.6 & 82 & 81.7 & 0.8 \\
\hline \multicolumn{6}{|l|}{ Laboratory parameters } \\
\hline UACR (mg/g) & 57 & $80.2(41.4-359.4)$ & 55 & $6.0(0-15.8)$ & $<0.001$ \\
\hline Creatinine $(\mu \mathrm{mol} / \mathrm{L})$ & 67 & $62(53-97)$ & 82 & $71(53-80)$ & 0.8 \\
\hline eGFR by MDRD equation (mL/min) & 64 & $142 \pm 70$ & 76 & $138 \pm 44$ & 0.7 \\
\hline $\operatorname{TRV}(\mathrm{m} / \mathrm{s})$ & 45 & $2.5(2.2-3.0)$ & 50 & $2.4(2.1-2.8)$ & 0.1 \\
\hline Hemoglobin $(g / L)$ & 67 & $88 \pm 18$ & 82 & $93 \pm 17$ & 0.05 \\
\hline Percentage reticulocyte ${ }^{\mathrm{a}}(\%)$ & 65 & $7.0(4.7-10.1)$ & 82 & $5.5(3.7-8.1)$ & 0.009 \\
\hline Fetal hemoglobin ${ }^{\mathrm{a}}(\%)$ & 61 & $5.9(3.0-9.3)$ & 77 & $6.1(2.6-14.5)$ & 0.6 \\
\hline Lactate dehydrogenase (U/L) & 60 & $932(681-1364)$ & 79 & $802(652-1104)$ & 0.06 \\
\hline Total bilirubin level $(\mu \mathrm{mol} / \mathrm{L})$ & 60 & $55(19-51)$ & 81 & $29(17-44)$ & 0.06 \\
\hline
\end{tabular}

Parametric continuous variables expressed as mean \pm standard deviation; non-parametric continuous variables expressed as median (inter-quartile range).

ACEi, angiotensin-converting enzyme inhibitor; ARB, angiotensin receptor blocker; NSAID, non-steroidal anti-inflammatory drug; TRV, tricuspid regurgitation jet velocity; UACR, urinary albumin-to-creatinine ratio; eGFR, estimated glomerular filtration rate; MDRD, Modification of Diet in Renal Disease.

${ }^{\mathrm{a}}$ Continuous variable

\section{DISCUSSION}

In this large cohort of adult sickle cell patients, we found hydroxyurea to be associated with a significantly lower prevalence of albuminuria. Moreover, this association is independent from potential variables affecting proteinuria, namely age, ACEi/ARB use, TRV, systemic hypertension or history of acute chest syndrome. Our findings raise questions about both the potential mechanism by which hydroxyurea may provide renal benefit to SCD patients and about the implications for its use specifically for this reason.

Several potential mechanisms exist by which hydroxyurea could affect proteinuria. Indeed, hydroxyurea is known to increase fetal hemoglobin, alter expression of adhesion molecules, decrease reticulocyte and neutrophil counts and enhance local nitric oxide generation $[12,28]$. An obvious mechanism is indirect, by reducing the ischemic renal injury via a decline in sickling occurrence and reduction in hemolysis. Numerous markers of hemolysis have been reported to be associated with increased urinary albumin excretion [29]. In our own cohort, patients without albuminuria demonstrated a higher hemoglobin level as well as a trend toward lower lactate dehydrogenase and bilirubin levels suggesting less hemolysis in these patients. Whether decreased hemolysis is the primary mechanism by which hydroxyurea is affecting albuminuria remains uncertain. Our study showed no evidence of a direct association between makers of hemolysis and use of hydroxyurea. It could be explained partially by indications of hydroxyurea therapy, which was frequently started in the context of recurrent painful episodes. However, there might be a potential role of hydroxyurea on hemolysis from our data despite no clear direct association. Indeed, patients unexposed to hydroxyurea with albuminuria had more hemolysis compared with untreated patients without albuminuria, with a significantly higher median reticulocyte percent, serum lactate dehydrogenase and total bilirubin. In other words, hydroxyurea may decrease hemolysis in patients with albuminuria, making a significant difference between treated and untreated patients undetectable. Fetal hemoglobin level and albuminuria were not directly related in our analyses, although we observed a clear increase in fetal hemoglobin in patients on hydroxyurea therapy, regardless of their albuminuria status. This certainly reflects the expected 'therapeutic' effect from the drug. In our primary multivariate model, we purposely withheld inclusion hemolysis markers as they were likely causal intermediates. With lactate dehydrogenase added to our secondary logistic regression model, we produced a partial loss of the detectable influence of hydroxyurea on albuminuria although we still noted a trend toward a protective effect of hydroxyurea. This 


\begin{tabular}{|c|c|c|c|c|c|}
\hline & $n$ & Hydroxyurea & $n$ & No hydroxyurea & $\mathrm{P}$ \\
\hline Microalbuminuria $^{\mathrm{a}}(\%)$ & 55 & 27.3 & 57 & 42.1 & 0.1 \\
\hline Macroalbuminuria $^{\mathrm{a}}(\%)$ & 55 & 10.9 & 57 & 21.1 & 0.1 \\
\hline Presence of albuminuria (\%) & 75 & 34.7 & 74 & 55.4 & 0.01 \\
\hline \multicolumn{6}{|l|}{ Clinical parameters } \\
\hline Age (years) & 75 & $36(28-48)$ & 74 & $40(28-49)$ & 0.6 \\
\hline Sex $(\%)$ & 75 & & 74 & & 0.5 \\
\hline $\mathrm{F}$ & & 61.3 & & 66.2 & \\
\hline M & & 38.7 & & 33.8 & \\
\hline Genotype (\%) & 75 & & 74 & & $<0.001$ \\
\hline HbSS & & 92 & & 59.5 & \\
\hline $\mathrm{HbS} \beta^{0}$ & & 4 & & 9.5 & \\
\hline $\mathrm{HbS}^{+}$ & & 1.3 & & 8.1 & \\
\hline HbSC & & 2.7 & & 21.6 & \\
\hline HbSD & & 0 & & 1.4 & \\
\hline ACEi/ARB use (\%) & 75 & 14.7 & 74 & 17.6 & 0.6 \\
\hline NSAID use (\%) & 75 & 40 & 74 & 35.1 & 0.5 \\
\hline Hypertension (\%) & 75 & 22.7 & 74 & 31.1 & 0.2 \\
\hline Systolic blood pressure (mmHg) & 75 & $122 \pm 17$ & 74 & $122 \pm 16$ & 0.8 \\
\hline Diastolic blood pressure (mmHg) & 75 & $71 \pm 12$ & 74 & $70 \pm 11$ & 0.6 \\
\hline Diabetes $(\%)$ & 75 & 6.7 & 74 & 4.1 & 0.7 \\
\hline $\mathrm{TRV} \geq 2.5 \mathrm{~m} / \mathrm{s}(\%)$ & 46 & 37 & 49 & 57.1 & 0.05 \\
\hline Acute chest syndrome (\%) & 75 & 94.7 & 74 & 70.3 & $<0.001$ \\
\hline \multicolumn{6}{|l|}{ Laboratory parameters } \\
\hline UACR (mg/g) & 55 & $17.9(6.0-53.0)$ & 57 & $40.5(7.0-204.9)$ & 0.04 \\
\hline Creatinine $(\mu \mathrm{mol} / \mathrm{L})$ & 75 & $62(53-80)$ & 74 & $71(53-97)$ & 0.1 \\
\hline eGFR by MDRD equation ( $\mathrm{mL} / \mathrm{min})$ & 70 & $151 \pm 55$ & 70 & $128 \pm 58$ & 0.02 \\
\hline $\mathrm{TRV}(\mathrm{m} / \mathrm{s})$ & 46 & $2.3(2.1-2.7)$ & 49 & $2.5(2.3-3.0)$ & 0.009 \\
\hline Hemoglobin $(g / L)$ & 75 & $91 \pm 19$ & 74 & $90 \pm 16$ & 0.8 \\
\hline Percentage reticulocyte $^{\mathrm{b}}(\%)$ & 74 & $6.0(4.3-8.0)$ & 73 & $6.6(4.2-9.8)$ & 0.4 \\
\hline Fetal hemoglobin ${ }^{\mathrm{b}}(\%)$ & 69 & $8.8(5.2-15.6)$ & 69 & $3.2(1.4-7.1)$ & $<0.001$ \\
\hline Lactate dehydrogenase (U/L) & 71 & 809 (664-1119) & 68 & $916(671-1385)$ & 0.1 \\
\hline Total bilirubin level $(\mu \mathrm{mol} / \mathrm{L})$ & 72 & $31(17-41)$ & 69 & $38(17-56)$ & 0.1 \\
\hline
\end{tabular}

Parametric continuous variables expressed as mean \pm standard deviation; non-parametric continuous variables expressed as median (inter-quartile range).

ACEi, angiotensin-converting enzyme inhibitor; ARB, angiotensin receptor blocker; NSAID, non-steroidal anti-inflammatory drug; TRV, tricuspid regurgitation jet velocity; UACR, urinary albumin-to-creatinine ratio; eGFR, estimated glomerular filtration rate; MDRD, Modification of Diet in Renal Disease.

${ }^{\mathrm{a}}$ Microalbuminuria and macroalbuminuria assessment was performed in 112 patients with documented urinary albumin-to-creatinine ratio but not in patients with documented dipstick only.

${ }^{\mathrm{b}}$ Continuous variable.

persistence suggests other potentially direct renal mechanisms by which hydroxyurea might influence albuminuria.

One alternative mechanism could be mediated directly through the release of nitric oxide, offsetting vasoconstrictive effects of free heme-related nitric oxide scavenging $[3,30]$. The release of nitric oxide may also abrogate endothelial dysfunction, which has been suggested to play a role in albuminuria [20]. Another effect may be upon podocytes themselves. SCD nephropathy has been linked to FSGS, often the perihilar variant, found in glomerulopathies implying adaptive changes to hyperfiltration $[3,5,31]$. Proliferative variants of FSGS, 'collapsing’ or cellular, are marked by altered expression of proliferation markers such as cyclin D1. These result in a dysregulated podocyte phenotype characterized by dedifferentiation and proliferation [32]. Hydroxyurea may destabilize cyclin D1 thus reducing podocyte proliferation [33].

Few treatment options specifically for SCD nephropathy are described in the literature. Only ACEi were demonstrated to reduce proteinuria in few studies with relatively short follow-up $[4,5,10,11]$. Use of ACEi in SCD patients is, however, limited by the presence of concomitant hyperkalemia
Table 4. Univariate determinants of albuminuria

\begin{tabular}{lll}
\hline & $\begin{array}{l}\text { Odds ratio } \\
(95 \% \mathrm{CI})\end{array}$ & P-value \\
\hline Age & $1.01(0.981-1.032)$ & 0.6 \\
Hydroxyurea use & $0.43(0.221-0.827)$ & 0.01 \\
ACEi/ARB use & $3.64(1.409-9.421)$ & 0.008 \\
TRV & $1.69(0.741-3.852)$ & 0.2 \\
Hypertension & $1.51(0.731-3.134)$ & 0.3 \\
History of acute chest syndrome & $1.14(0.485-2.680)$ & 0.8 \\
Lactate dehydrogenase & $1.00(1.000-1.002)$ & 0.03 \\
\hline
\end{tabular}

ACEi, angiotensin-converting enzyme inhibitor; ARB, angiotensin receptor blocker; TRV, tricuspid regurgitation jet velocity.

and may be associated with a further decline of renal function in patients with an already low GFR [7]. Hydroxyurea is generally used in severe SCD to reduce the occurrence of vasoocclusive crises thereby diminishing morbidity and mortality [13]. Currently, albuminuria and overt nephropathy by themselves are not indications for hydroxyurea therapy in SCD. In this cohort, patients on hydroxyurea had features of more 
Table 5. Multivariate determinants of albuminuria

\begin{tabular}{|c|c|c|c|c|}
\hline & \multicolumn{2}{|l|}{ Model 1} & \multicolumn{2}{|l|}{ Model $2^{\mathrm{a}}$} \\
\hline & $\begin{array}{l}\text { Odds ratio } \\
\text { (95\% confidence interval) }\end{array}$ & P-value & $\begin{array}{l}\text { Odds ratio } \\
\text { ( } 95 \% \text { confidence interval) }\end{array}$ & P-value \\
\hline Hydroxyurea use & $0.28(0.11-0.75)$ & 0.01 & $0.31(0.12-0.80)$ & 0.02 \\
\hline ACEi/ARB use & $9.13(1.33-62.83)$ & 0.03 & $4.58(0.97-21.5)$ & 0.05 \\
\hline TRV & $0.84(0.27-2.57)$ & 0.8 & $0.78(0.28-2.17)$ & 0.6 \\
\hline Hypertension & $0.39(0.09-1.77)$ & 0.2 & $\mathrm{n} / \mathrm{a}$ & \\
\hline
\end{tabular}

$\mathrm{ACEi}$, angiotensin-converting enzyme inhibitor; ARB, angiotensin receptor blocker; TRV, tricuspid regurgitation jet velocity; n/a, not applicable.

${ }^{a}$ Model 2: simplified model, with removal of covariates via backward elimination and change-in-estimate testing of $<10 \%$.

severe SCD (such as acute chest syndrome) likely relating to an indication for the use of this drug. While more severe SCD would typically be associated with albuminuria [8], we noted reduced prevalence in those receiving hydroxyurea, despite having an otherwise more severe phenotype.

Very small case series have suggested a short-term beneficial effect of hydroxyurea on proteinuria reduction, with similar trends noted in retrospective and prospective pediatric studies [16-19, 34-37]. Small prospective series in pediatric populations (a total of 20 patients) have shown an improvement in proteinuria after commencing hydroxyurea [16-18]. Some of these demonstrated this effect to be beyond that achieved by an ACEi or ARB alone [16]. Our own data demonstrate that even after adjusting for these agents, the benefit of hydroxyurea persisted. Larger cross-sectional studies initially designed to describe the prevalence of albuminuria have failed to demonstrate a correlation between albuminuria and hydroxyurea use $[35,38]$. This finding may be partially explained by the small number of patients on hydroxyurea and because those cohorts were composed of pediatric patients with a lower prevalence of albuminuria as found in adults with SCD.

The Pediatric Hydroxyurea Phase III Clinical (BABY HUG) Trial reported the influence of hydroxyurea therapy in 193 infants and evaluated several renal outcomes such as GFR, urine osmolality and kidney size but did not observe a substantial benefit $[34,39]$. In contradistinction, our study revealed an association between hydroxyurea and a separate renal outcome - urinary protein excretion. This outcome was not evaluated in BABY HUG likely due to the low prevalence of albuminuria and the difficulty of obtaining urine samples in such a young population. An observable benefit of hydroxyurea in our population is likely attributable to the fact that our population was composed exclusively of older adult patients more likely to have end-organ damage, lending toward a higher prevalence of albuminuria. A beneficial renal effect of hydroxyurea may also only be demonstrable in patients who are of sufficient age to exhibit more substantial renal disease, i.e. proteinuria or reduced eGFR. The same rationale can be applied to a recently published study in which a trend toward lower microalbuminuria was observed in children under therapy with hydroxyurea [37].

Our findings must be viewed in the context of several limitations, mainly related to their retrospective and cross-sectional nature. Most importantly, we are unable to discern the longitudinal effect of hydroxyurea on commonly accepted definitive renal outcomes such as loss of eGFR or end-stage renal disease. Urine dipstick testing was used to identify albuminuria in a subset of the population rather than direct measurement of UACR. Dipstick testing may be over-sensitive in patients with concentrated urine, but this would be less likely in SCD patients who frequently have a concentrating defect. We conservatively required two dipstick tests at this level to define albuminuria and used a cut-off that correlates well with UACR $\geq 30 \mathrm{mg} / \mathrm{g}$ in the general population [22]. Further, prior studies in sickle cell nephropathy have used $\geq 1+$ proteinuria by dipstick to identify patients with nephropathy [23]. Our definition of hydroxyurea use was defined as 3 months of use prior to albuminuria assessment. Prior use of hydroxyurea in some patients categorized as non-users could have longer lasting effects. However, we would expect this to lead to a lower prevalence of albuminuria in non-users making it less likely to observe the protective association of the drug. Similarly, having fewer patients of the HbSC genotype, a less severe SCD, on hydroxyurea should diminish our ability to demonstrate a benefit of hydroxyurea. Despite these limitations, we still detect a statistically significant reduction in albuminuria prevalence in hydroxyurea users. Finally, our study does not take into account any histological measures of renal disease severity specific to $S C D$ as suggested by Guasch et al. [7]. The small number of subjects with diabetes mellitus does reduce the possible bias of a concomitant glomerular injury that may affect the real prevalence of SCDrelated glomerulopathy.

Our results demonstrated a decreased prevalence of albuminuria in adult SCD patients on hydroxyurea. After controlling for several relevant clinical variables, the lower likelihood of albuminuria persisted among those patients taking hydroxyurea. Based upon our findings and those of prior case series, hydroxyurea may be beneficial for prevention of SCD nephropathy or delay of its progression as seen with ACEi/ARB in diabetes. This hypothesis can best be tested by a prospective randomized controlled clinical trial in a SCD population followed to an age in which albuminuria is of sufficient prevalence. Hydroxyurea is promising particularly due to its ready availability and relatively low cost, and our results at least provide further incentive for physicians' prescription of hydroxyurea in SCD patients and for patients' adherence when prescribed these agents. While our findings suggest that hydroxyurea may reduce the prevalence of albuminuria in 
SCD, further investigation is needed to determine whether it may prevent overt SCD progression to end-stage renal disease and whether SCD nephropathy alone should be an indication for hydroxyurea use.

\section{SUPPLEMENTARY DATA}

Supplementary data are available online at http://ndt.oxfordjournals.org.

\section{ACKNOWLEDGEMENTS}

The authors thank Susan K. Jones for her help in data management. L.-P.L. received salary support from Hôpital MaisonneuveRosemont Scholarship of Improvement Program, Société Québécoise de Néphrologie and Department of Medicine, Université de Montréal. V.K.D. received support from Duke-UNC Clinical Hematology Research Career Development program (NIH/NHLBI K12HL087097-05, PI: Telen) and NIH/NHLBI 1R01HL111659-01, PI: Ataga.

\section{CONFLICT OF INTEREST STATEMENT}

None of the authors have any competing interests. The results presented here have not been published previously in whole or in part, except in abstract form.

\section{REFERENCES}

1. Bunn HF. Pathogenesis and treatment of sickle cell disease. N Engl J Med 1997; 337: 762-769

2. Pham PT, Pham PC, Wilkinson AH et al. Renal abnormalities in sickle cell disease. Kidney Int 2000; 57: 1-8

3. Nath KA, Katusic ZS. Vasculature and kidney complications in sickle cell disease. J Am Soc Nephrol 2012; 23: 781-784

4. Ataga KI, Orringer EP. Renal abnormalities in sickle cell disease. Am J Hematol 2000; 63: 205-211

5. Falk RJ, Scheinman J, Phillips G et al. Prevalence and pathologic features of sickle cell nephropathy and response to inhibition of angiotensin-converting enzyme. N Engl J Med 1992; 326: 910-915

6. Guasch A, Navarrete J, Nass $\mathrm{K}$ et al. Glomerular involvement in adults with sickle cell hemoglobinopathies: Prevalence and clinical correlates of progressive renal failure. J Am Soc Nephrol 2006; 17: 2228-2235

7. Guasch A, Cua M, Mitch WE. Early detection and the course of glomerular injury in patients with sickle cell anemia. Kidney Int 1996; 49: 786-791

8. Powars DR, Elliott-Mills DD, Chan L et al. Chronic renal failure in sickle cell disease: risk factors, clinical course, and mortality. Ann Intern Med 1991; 115: 614-620

9. Powars DR, Chan LS, Hiti A et al. Outcome of sickle cell anemia: a 4decade observational study of 1056 patients. Medicine (Baltimore) 2005; 84: 363-376

10. Foucan L, Bourhis V, Bangou J et al. A randomized trial of captopril for microalbuminuria in normotensive adults with sickle cell anemia. Am J Med 1998; 104: 339-342

11. Aoki RY, Saad ST. Enalapril reduces the albuminuria of patients with sickle cell disease. Am J Med 1995; 98: 432-435

12. Charache S, Terrin ML, Moore RD et al.; Investigators of the Multicenter Study of Hydroxyurea in Sickle Cell Anemia. Effect of hydroxyurea on the frequency of painful crises in sickle cell anemia. N Engl J Med 1995; 332 $1317-1322$

13. Steinberg $\mathrm{MH}$, Barton $\mathrm{F}$, Castro $\mathrm{O}$ et al. Effect of hydroxyurea on mortality and morbidity in adult sickle cell anemia: risks and benefits up to 9 years of treatment. JAMA 2003; 289: 1645-1651

14. Steinberg MH, McCarthy WF, Castro O et al. The risks and benefits of long-term use of hydroxyurea in sickle cell anemia: A 17.5 year follow-up. Am J Hematol 2010; 85: 403-408

15. Voskaridou E, Christoulas D, Bilalis A et al. The effect of prolonged administration of hydroxyurea on morbidity and mortality in adult patients with sickle cell syndromes: results of a 17-year, single-center trial (LaSHS). Blood 2010; 115: 2354-2363

16. Fitzhugh CD, Wigfall DR, Ware RE. Enalapril and hydroxyurea therapy for children with sickle nephropathy. Pediatr Blood Cancer 2005; 45: 982-985

17. McKie KT, Hanevold CD, Hernandez C et al. Prevalence, prevention, and treatment of microalbuminuria and proteinuria in children with sickle cell disease. J Pediatr Hematol Oncol 2007; 29: 140-144

18. Alvarez O, Lopez-Mitnik G, Zilleruelo G. Short-term follow-up of patients with sickle cell disease and albuminuria. Pediatr Blood Cancer 2008; 50 : 1236-1239

19. Alvarez O, Montane B, Lopez G et al. Early blood transfusions protect against microalbuminuria in children with sickle cell disease. Pediatr Blood Cancer 2006; 47: 71-76

20. Ataga KI, Brittain JE, Moore D et al. Urinary albumin excretion is associated with pulmonary hypertension in sickle cell disease: potential role of soluble FMS-like tyrosine kinase-1. Eur J Haematol 2010; 85: 257-263

21. Steinmetz J, Henny J, Gueguen R. Determination of glucose, proteins, blood and leucocytes in urines: evaluation of automated analyzer Aution Max AX 4280. Ann Biol Clin (Paris) 2005; 63: 619-626

22. White SL, Yu R, Craig JC et al. Diagnostic accuracy of urine dipsticks for detection of albuminuria in the general community. Am J Kidney Dis 2011; 58: 19-28

23. Ashley-Koch AE, Okocha EC, Garrett ME et al. MYH9 and APOL1 are both associated with sickle cell disease nephropathy. Br J Haematol 2011; 155: 386-394

24. Levey AS, Bosch JP, Lewis JB et al. A more accurate method to estimate glomerular filtration rate from serum creatinine: a new prediction equation. Modification of Diet in Renal Disease Study Group. Ann Intern Med 1999; 130: 461-470

25. Yock PG, Popp RL. Noninvasive estimation of right ventricular systolic pressure by Doppler ultrasound in patients with tricuspid regurgitation. Circulation 1984; 70: 657-662

26. Gladwin MT, Sachdev V, Jison ML et al. Pulmonary hypertension as a risk factor for death in patients with sickle cell disease. N Engl J Med 2004; 350: 886-895

27. Howards PP, Schisterman EF, Poole C et al. 'Toward a clearer definition of confounding' revisited with directed acyclic graphs. Am J Epidemiol 2012; 176: 506-511

28. McGann PT, Ware RE. Hydroxyurea for sickle cell anemia: what have we learned and what questions still remain? Curr Opin Hematol 2011; 18 : 158-165

29. Day TG, Drasar ER, Fulford T et al. Association between hemolysis and albuminuria in adults with sickle cell anemia. Haematologica 2012; 97: 201-205

30. Kovacic P. Hydroxyurea (therapeutics and mechanism): metabolism, carbamoyl nitroso, nitroxyl, radicals, cell signaling and clinical applications. Med Hypotheses 2011; 76: 24-31

31. Becker AM. Sickle cell nephropathy: challenging the conventional wisdom. Pediatr Nephrol 2011; 26: 2099-2109

32. Albaqumi M, Barisoni L. Current views on collapsing glomerulopathy. J Am Soc Nephrol 2008; 19: 1276-1281

33. Mukherji A, Janbandhu VC, Kumar V. HBx protein modulates PI3K/Akt pathway to overcome genotoxic stress-induced destabilization of cyclin D1 and arrest of cell cycle. Indian J Biochem Biophys 2009; 46: 37-44

34. Alvarez O, Miller ST, Wang WC et al. Effect of hydroxyurea treatment on renal function parameters: results from the multi-center placebo-controlled BABY HUG clinical trial for infants with sickle cell anemia. Pediatr Blood Cancer 2012; 59: 668-674 
35. McPherson Yee M, Jabbar SF, Osunkwo I et al. Chronic kidney disease and albuminuria in children with sickle cell disease. Clin J Am Soc Nephrol 2011; 6: 2628-2633

36. Lebensburger J, Johnson SM, Askenazi DJ et al. Protective role of hemoglobin and fetal hemoglobin in early kidney disease for children with sickle cell anemia. Am J Hematol 2011; 86: 430-432

37. Aygun B, Mortier NA, Smeltzer MP et al. Hydroxyurea treatment decreases glomerular hyperfiltration in children with sickle cell anemia. Am J Hematol 2013; 88: 116-119
38. Becton LJ, Kalpatthi RV, Rackoff E et al. Prevalence and clinical correlates of microalbuminuria in children with sickle cell disease. Pediatr Nephrol 2010; 25: 1505-1511

39. Wang WC, Ware RE, Miller ST et al. Hydroxycarbamide in very young children with sickle-cell anaemia: a multicentre, randomised, controlled trial (BABY HUG). Lancet 2011; 377: 1663-1672

Received for publication: 22.2.2013; Accepted in revised form: 26.5.2013

\title{
The association between left ventricular global longitudinal strain, renal impairment and all-cause mortality
}

\author{
Rathika Krishnasamy ${ }^{1}$, Nicole M. Isbel ${ }^{1}$, Carmel M. Hawley ${ }^{1}$, Elaine M. Pascoe ${ }^{2}$, Rodel Leano ${ }^{3}$, \\ Brian A. Haluska ${ }^{3}$ and Tony Stanton ${ }^{3}$ \\ ${ }^{1}$ Department of Renal Medicine, University of Queensland at Princess Alexandra Hospital, Brisbane, Queensland, Australia, ${ }^{2}$ School of \\ Medicine, University of Queensland, Brisbane, Queensland, Australia and ${ }^{3}$ Cardiovascular Imaging Research Centre, School of Medicine, \\ University of Queensland at Princess Alexandra Hospital, Brisbane, Queensland, Australia
}

Correspondence and offprint requests to: Rathika Krishnasamy; E-mail: rathika.krishnasamy@health.qld.gov.au

\section{ABSTRACT}

Background. Left ventricular (LV) systolic dysfunction is an important predictor of cardiovascular death. Global longitudinal strain (GLS) is a widely available echocardiographic technique proven to be more sensitive than conventional ejection fraction (EF) in detecting subtle changes in LV function. However, the prognostic value of GLS in patients with chronic kidney disease (CKD) is unknown.

Methods. We studied 447 patients from a single center who were stratified according to estimated glomerular filtration rate (eGFR). GLS was calculated using two-dimensional speckle tracking and EF was measured using Simpson's biplane. Cox proportional hazard model was used to identify independent predictors of survival and measures of discrimination and reclassification were used to assess the predictive value of GLS. Multivariable regression models were used to evaluate clinical and laboratory factors associated with GLS.

Results. The mean EF was $58 \pm 11 \%$ and GLS was $-16.6 \pm 4.2 \%$. eGFR correlated negatively with GLS $(r=-0.14$, $\mathrm{P}=0.004$ ). Factors that were independently associated with GLS include gender, previous myocardial infarction, eGFR and phosphate $\left(R^{2}=0.16, \mathrm{P}<0.001\right)$. Sixty-four patients died in a follow-up of $5.2 \pm 1.4$ years. GLS remained a significant predictor of all-cause mortality [hazard ratio (HR) 1.08, 95\% confidence interval (CI) 1.01-1.15] following adjustment for age, diabetes mellitus, hypertension, eGFR and left ventricular mass index (LVMI). The strength of association between demographic data, eGFR, LVMI and mortality increased following addition of GLS [c-statistic 0.68 (95\% CI 0.61-0.74) to 0.71 (95\% CI 0.64-0.77), $\mathrm{P}=0.04]$. Addition of GLS also demonstrated a $21 \%$ net reclassification improvement in risk prediction for all-cause mortality over clinical factors.

Conclusions. GLS is an important predictor of all-cause mortality in CKD patients. Traditional and non-traditional risk factors such as phosphate are important determinants of GLS. Strain assessment in CKD patients may provide greater cardiovascular risk stratification.

Keywords: all-cause mortality, chronic kidney disease, ejection fraction, global longitudinal strain

\section{INTRODUCTION}

Cardiovascular disease (CVD) is the leading cause of death in patients with chronic kidney disease (CKD) [1]. There is a 